ORIGINAL ARTICLE

\title{
A shift to a peripheral Th2-type cytokine pattern during the carcinogenesis of cervical cancer becomes manifest in CIN III lesions
}

\author{
A G Bais, I Beckmann, J Lindemans, P C Ewing, C J L M Meijer, P J F Snijders, T J M Helmerhorst
}

J Clin Pathol 2005;58:1096-1100. doi: 10.1136/jicp.2004.025072

See end of article for authors' affiliations

Correspondence to:

ProfessorTJMHelmerhorst, Department of Obstetrics and Gynaecology,

Erasmus University

Medical Centre Rotterdam, PO Box 2040, 3000 CA, Rotterdam, The

Netherlands; t.

helmerhorst@erasmusmc. $\mathrm{nl}$

Accepted for publication 22 February 2005
Background: A shifted balance between T helper 1 (Th1)-type and Th2-type cytokines has been hypothesised in cervical dysplasia

Aims: To evaluate possible deregulation of the cytokine network by estimating the expression of peripheral cytokines in different stages of cervical disease and in relation to the presence or absence of high risk human papillomavirus (HR-HPV).

Methods: Twenty one HR-HPV positive women with high grade cervical intraepithelial neoplasia (CIN II-III) and 12 patients with invasive cervical carcinoma formed the study groups. Two control groups consisted of 10 HR-HPV positive and 11 HR-HPV negative women without CIN. Differences in leucocyte subgroups were evaluated by a differential leucocyte count. Plasma concentrations of tumour necrosis factor $\alpha$ (TNF $\alpha$ ), TNF $\alpha$ receptors TNFRI and TNFRII, interferon $\gamma$ (IFN $\gamma$ ), interleukin 2 (IL-2), IL-12, IL-4, and IL-10 were determined by enzyme linked immunosorbent assays.

Results: Leucocyte counts in patients with CIN III and carcinoma were significantly higher than in controls. Plasma IFN $\gamma$ concentrations were significantly lower in patients with CIN III and carcinoma than in women with CIN II or controls. Plasma concentrations of IL-12, IL-2, IL-4, and TNF $\alpha$ did not differ significantly between groups, but significantly lower plasma concentrations of TNFRII were found in CIN III and carcinoma compared with CIN II. IL-10 was detected with increased frequency in the plasma of patients with CIN III and carcinoma.

Conclusions: These results indicate that a shift to a Th2-type cytokine pattern during the carcinogenesis of cervical cancer occurs in women with CIN III lesions.
1 is well established that high risk (HR) human papillomavirus (HPV) types can cause the development of cervical cancer.$^{1-3}$ Most HPV infections are cleared without further consequences for the host, but a certain proportion of untreated infections with HR-HPV types may give rise to high grade cervical intraepithelial neoplasia (CIN II-III) and cervical cancer. ${ }^{4-6}$ There is evidence that cell mediated immune responses of the host, both systemic and local, are important determinants of the course of infection. ${ }^{7}$

"The immune response is mediated through the release of different cytokines, which can influence the synthesis and actions of one another in the setting of an immunoregulating cytokine network"

Cell mediated immune responses are regulated by $\mathrm{T}$ cells ( $\mathrm{T}$ helper (Th) cells and cytotoxic lymphocytes) in cooperation with antigen presenting cells. The immune response is mediated through the release of different cytokines, which can influence the synthesis and actions of one another in the setting of an immunoregulating cytokine network. Cytokines in immune responses to infection are often classified as immunostimulating (tumour suppressing) Thl-type cytokines such as interferon $\gamma$ (IFN $\gamma)$, tumour necrosis factor $\alpha$ (TNF $\alpha$ ), interleukin 2 (IL-2), and IL-12, which mainly induce cell mediated immunity, and Th2-type cytokines (IL-4, IL-5, IL-6, IL-8, and IL-10), which are immunoinhibitory for cell mediated responses and predominantly induce humoral immunity. ${ }^{89}$ Qualitative and quantitative analyses of Thltype and Th2-type cytokine profiles have been used to determine the immune response in several human diseases, including HPV associated CIN. ${ }^{10}{ }^{11}$ Studies describing circulating cytokines in the plasma of patients with cervical dysplasia or cancer are either scarce, deal with one or only a few cytokines, or are contradictory and only incidentally related to HR-HPV. ${ }^{12-20}$ In our present study, we analysed changes in the peripheral cytokine pattern in HR-HPV positive women with different stages of CIN compared with HR-HPV positive and negative controls.

\section{MATERIALS AND METHODS}

Patients and controls

Women with abnormal cervical cytology referred to the outpatient clinic of the gynaecology department of the Erasmus University Medical Centre Rotterdam, the Netherlands between July 2000 and August 2002 were selected as the study group. HPV sampling and a cervical biopsy were obtained from all participating women. Histology results were defined as mild dysplasia (CIN I), moderate dysplasia (CIN II), severe dysplasia (CIN III), or (micro)invasive cancer. An experienced pathologist revised all histological samples. Our selection of study patients was based on the presence of HR-HPV and the severity of CIN. Women with CIN I lesions (mild dysplasia) were excluded because more than $50 \%$ of our patients with CIN I were HRHPV negative. Healthy women who entered the outpatient clinic for a regular sterilisation procedure were recruited as

Abbreviations: CIN, cervical intraepithelial neoplasia; CV, coefficient of variation; HPV, human papillomavirus; HR, high risk; IFN $\gamma$, interferon $\gamma$; IL, interleukin; MDC, minimal detectable concentration; PCR, polymerase chain reaction; s, soluble; Th, Thelper; TNF $\alpha$, tumour necrosis factor $\alpha$; TNFR, tumour necrosis factor $\alpha$ receptor 
Table 1 HPV typing in patients with cervical dysplasia (CIN II, CIN III, and carcinoma) and in HR-HPV positive controls

\begin{tabular}{llll}
\hline Controls $\mathbf{n = 1 0}$ & $\mathbf{C I N}$ II $\mathbf{n = 1 0}$ & $\mathrm{CIN}$ III $\mathbf{n = 1 1}$ & Carcinoma $\mathbf{n = 1 2}$ \\
\hline HR-HPV type & HR-HPV type & HR-HPV type & HR-HPV type \\
31 & 31 & 31,56 & 16 \\
31 & 31 & 31,18 & 16 \\
31 & 16 & 16 & 16 \\
$31,51,56$ & 16 & 16 & 16 \\
56 & 16 & 16 & 18 \\
$59,58,56$ & 16 & 16 & 18 \\
59 & $16,45,56$ & 16 & 18 \\
35,56 & 18,39 & 16 & 18 \\
52 & 39 & 16 & 18 \\
16 & 52 & $16(70)$ & 33 \\
& & 33 & $(70)$ \\
& & & 82
\end{tabular}

$\mathrm{CIN}$, cervical intraepithelial neoplasia; HPV, human papillomavirus; HR, high risk.

controls. Cervical cytology, histology, and HPV sampling were obtained. Exclusion criteria for all participants were: postmenopausal state, pregnancy at time of sampling, chronic diseases (diabetes, allergy, or autoimmune disease), signs of acute infection at the time of sampling, and an immunocompromised state. Two women with allergy, one woman with an autoimmune disease, and one woman with an acute infection at the time of sampling were excluded. With the exception of oral contraceptives, patients did not use medication on a regular base. Pain medication (non-steroidal anti-inflammatory drugs) was omitted for at least two weeks before sampling.

Our study protocol was approved by the hospital and university ethics committee and all women voluntarily gave signed informed consent.

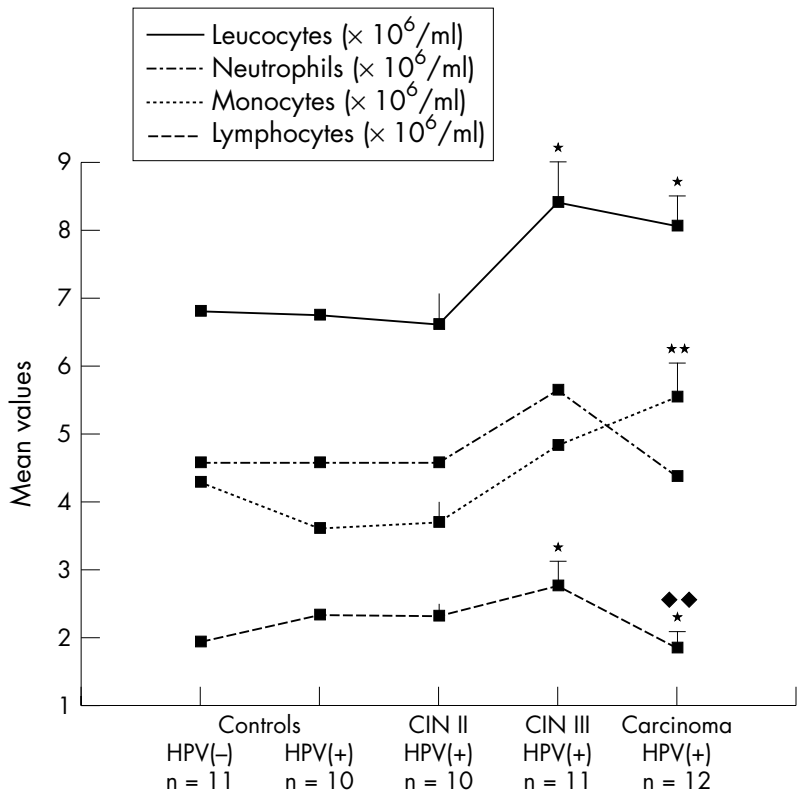

Figure 1 Differential white blood cell counts in patients with high risk human papillomavirus infection and cervical dysplasia and in high risk HPV negative and high risk HPV positive controls. Data are shown as mean \pm SEM. ${ }^{*} \mathrm{p} \leqslant 0.05$ when compared with $\mathrm{CIN} \mathrm{II;}{ }^{* *} \mathrm{p}<0.01$ when compared with CIN II. $\bullet p<0.01$ when compared with CIN III. CIN, cervical intraepithelial neoplasia; HPV, human papillomavirus.

\section{HPV sampling and determination}

Cervical scrapes for HPV detection and typing were taken by a cervical biosampler (Accellon Combi ${ }^{\circledR}$; Medscand Medical, Malmo, Sweden). HPV testing was performed with the consensus GP5+/GP6+ polymerase chain reaction (PCR) enzyme immunoassay for 37 (sub)types, as described previously. ${ }^{21}$ This test is clinically validated. ${ }^{22}$ We used $\beta$ globin PCR to identify sampling errors and to monitor for PCR inhibitors. In addition, reverse line blot analysis was performed on PCR enzyme immunoassay positive cases to identify individual HPV types.

\section{Blood sampling and processing}

Peripheral venous blood samples were collected between 8.00 and 12.00 a.m. in sterile endotoxin free vacutainers (Becton Dickinson, Meylan, France) with ethylenediaminetetraacetic acid (EDTA) as anticoagulant and immediately centrifuged at $1500 \times \mathrm{g}$ for 10 minutes at $4^{\circ} \mathrm{C}$. Plasma samples were stored at $-80^{\circ} \mathrm{C}$ until assayed.

A differential leucocyte count was performed in all blood samples with a Sysmex XE-2100.

\section{Cytokine determinations}

All plasma samples were analysed by commercially available enzyme linked immunoassays (Biosource Europe, Nivelle, Belgium) for the cytokines $\mathrm{TNF} \alpha$, soluble $\mathrm{TNF} \alpha$ receptors (sTNFRI and sTNFRII), IFN $\gamma$, IL-2, IL-4, IL-10, and IL- $12 .{ }^{23}$ The detecting antibody in the immunoassay for IL-12 recognised the bioactive heterodimeric (p40 + p35) cytokine, in addition to the p40 subunit homodimer. According to the manufacturer, the minimal detectable concentrations (MDCs) and intra-assay and interassay coefficients of variation (CVs) were as follows: TNF $\alpha$ : MDC, $3 \mathrm{pg} / \mathrm{ml}$; CVs, $<6 \%$ and $<10 \%$; sTNFRI: MDC, $50 \mathrm{pg} / \mathrm{ml}$; CVs, $<7 \%$ and $<10 \%$; sTNFRII: MDC, $100 \mathrm{pg} / \mathrm{ml}$; CVs, $<7 \%$ and $<10 \%$; IFN $\gamma$ : MDC, $2 \mathrm{pg} / \mathrm{ml}$; CVs, $<5 \%$ and $<10 \%$; IL-2: MDC, $7 \mathrm{pg} / \mathrm{ml}$; CVs, $<6 \%$ and $<10 \%$; IL-4: MDC, $2 \mathrm{pg} / \mathrm{ml}$; CVs, $<5 \%$ and $<7 \%$; IL- 10 : MDC, $1 \mathrm{pg} / \mathrm{ml} ; \mathrm{CVs}, \quad<5 \%$ and $<10 \%$; IL- $12+$ p40: MDC, $1.5 \mathrm{pg} / \mathrm{ml} ; \quad \mathrm{CVs}, \quad<10 \%$ and $<10 \%$

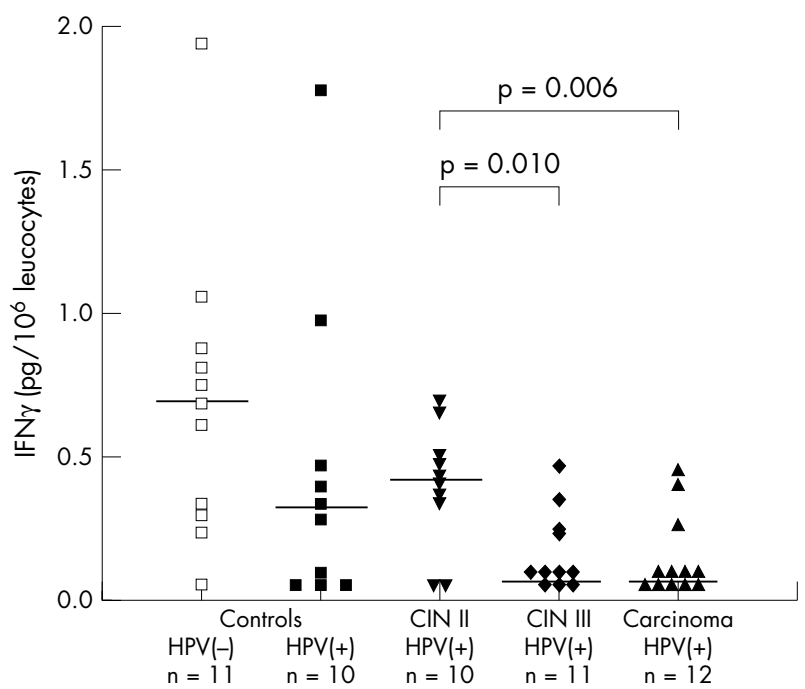

Figure 2 Plasma concentrations of IFN- $\gamma$ in high risk HPV positive patients with cervical dysplasia and in high risk HPV negative and high risk HPV positive controls. Median values are indicated by a horizontal line. CIN, cervical intraepithelial neoplasia; HPV, human papillomavirus; IFN $\gamma$, interferon $\gamma$. 


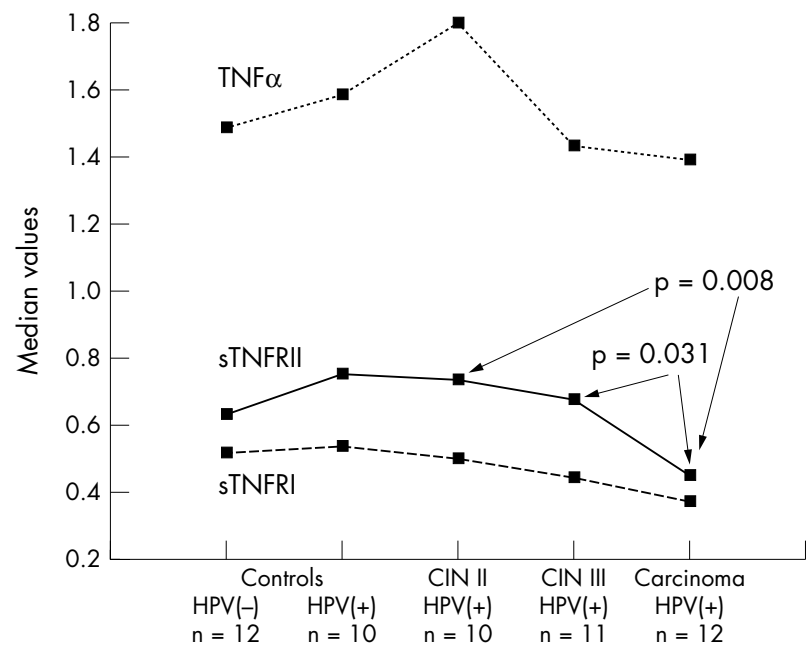

Figure 3 Plasma concentrations of TNF $\alpha$ and its soluble receptors sTNFRI and sTNFRII in high risk HPV positive patients with cervical dysplasia and in HPV negative and HPV positive controls. Median concentrations were: TNF $\alpha, 10^{6} \mathrm{pg} / \mathrm{ml} ;$ sTNFRI, $10^{6} \mathrm{ng} / \mathrm{ml}$; and sTNFRII, $10^{6} \mathrm{ng} / \mathrm{ml}$. CIN, cervical intraepithelial neoplasia; HPV, human papillomavirus; TNF $\alpha$, tumour necrosis factor $\alpha$.

To calculate cytokine ratios, values below the detection limit were assigned concentrations equal to one half of the lower detection limit of each assay.

\section{Statistical analysis}

Cytokine data are presented as medians with ranges unless stated otherwise. The non-parametric Kruskal-Wallis test and Mann-Whitney $U$ test were used as appropriate to assess differences in cytokine values between groups, and Spearman's rank test was used to investigate correlations between cytokine variables. A two tailed p value of 0.05 was chosen to represent significance. Differences in leucocytes and leucocyte subpopulations were evaluated by unpaired two tailed $t$ tests.

\section{RESULTS}

\section{The study groups}

Our study group comprised 33 women: 10 women with moderate dysplasia (CIN II), 11 women with severe dysplasia (CIN III), and 12 women with cervical carcinoma (10 squamous cell carcinomas, two adenocarcinomas). The control groups consisted of 11 women without cervical dysplasia without HR-HPV and 10 women without cervical dysplasia but with a positive HR-HPV test. The mean (SD) age was 32.4 (6.2) years for the HR-HPV negative controls, 27.9 (7.4) years for the HR-HPV positive controls, 33.4 (5.7) years for patients with CIN II, 32.3 (5.5) years for those with
CIN III, and 35.6 (7.2) years for women with invasive carcinoma.

\section{Results of HPV determinations}

HPV types 16, 18, and 31 were the most frequently detected HR-HPV types ( $\mathrm{n}=18, \mathrm{n}=7$, and $\mathrm{n}=8$, respectively). Other HR types were less common: HPV-33, $\mathrm{n}=2$; HPV-35, $\mathrm{n}=1$; HPV-39, $\mathrm{n}=2$; HPV-45, $\mathrm{n}=1$; HPV-51, $\mathrm{n}=1$; HPV-52, $\mathrm{n}=2$; HPV-56, $\mathrm{n}=6$; HPV-58, $\mathrm{n}=1$; HPV-59, $\mathrm{n}=2$; and HPV-82, $\mathrm{n}=1$. HPV-70 (low risk type) was detected together with HPV-16 in one woman with CIN III and as the single infecting agent in one of 12 patients with carcinoma. There were 34 single and eight multiple HPV infections. Table 1 summarises the HPV types within the study groups.

\section{The differential white blood cell count}

The total leucocyte count was higher in patients with CIN III and carcinoma than in CIN II and the control groups; the difference between CIN II and CIN III was significant. The neutrophil count showed a similar course, with a significant increase from CIN II to carcinoma. Absolute monocyte and lymphocyte counts varied significantly between groups, being highest in women with CIN III. Figure 1 shows the results of the differential white blood cell counts.

\section{The cytokine profile}

The results of cytokine assays were calculated for each $10^{6}$ leucocytes, to stratify for possible different numbers of cytokine producing leucocytes between study subjects.

Analysis of the peripheral cytokine profile in our study groups revealed different courses for each of the eight investigated cytokines. Differences between groups were seen for IFN $\gamma$ concentrations, with a significant decrease from CIN II to CIN III and carcinoma $(p \leqslant 0.01)$, as summarised in fig 2.

There were no significant differences in plasma concentrations of TNF $\alpha$ between the control and study groups. Both TNF receptors were significantly correlated in all groups $(r=0.973, \mathrm{p}=0.000$ for HR-HPV negative controls; $r=0.855, \mathrm{p}=0.002$ for HR-HPV positive controls; $r=0.979, \mathrm{p}=0.001$ for patients with CIN II; $r=0.791$, $\mathrm{p}=0.004$ for patients with CIN III; and $r=0.748$, $\mathrm{p}=0.005$ for patients with invasive carcinoma). TNFRII values were highest in HR-HPV positive controls. A significant decrease was seen in patients with cervical carcinoma compared with patients with CIN II. A similar pattern was seen for TNFRI but the differences did not reach significance. Figure 3 shows the median plasma concentrations of TNF $\alpha$ and its two soluble receptors sTNFRI and sTNFRII in all study groups.

There was a significant increase $(p=0.037)$ in plasma concentrations of IL-2 between HR-HPV negative and HRHPV positive controls, but no other significant changes in IL2 values. IL-12 showed no significant differences between

Table 2 Plasma concentrations of IL-4 and IL-10 in HR-HPV positive patients with cervical dysplasia and in HR-HPV negative and positive controls

\begin{tabular}{llll}
\hline Group & $\mathbf{N}$ & $\begin{array}{l}\text { IL-4 } \\
\mathbf{x} / \mathbf{N} \text { plasmas }\end{array}$ & $\begin{array}{l}\text { detection limit* } \\
\mathbf{x} / \mathbf{N} \text { plasmas }\end{array}$ \\
\hline HR-HPV- controls & 11 & $2 / 11$ & $2 / 11$ \\
HR-HPV+ controls & 10 & $1 / 10$ & $0 / 10$ \\
HR-HPV+ CIN II & 10 & $2 / 10$ & $0 / 10$ \\
HR-HPV+ CIN III & 11 & $1 / 11$ & $4 / 11$ \\
HR-HPV+ carcinoma & 12 & $3 / 12$ & $7 / 12$ \\
\hline
\end{tabular}

*Detection limit for IL-4, $2 \mathrm{pg} / \mathrm{ml}$; detection limit for IL-10, $1 \mathrm{pg} / \mathrm{ml}$.

CIN, cervical intraepithelial neoplasia; HPV, human papillomavirus; HR, high risk; IL, interleukin. 


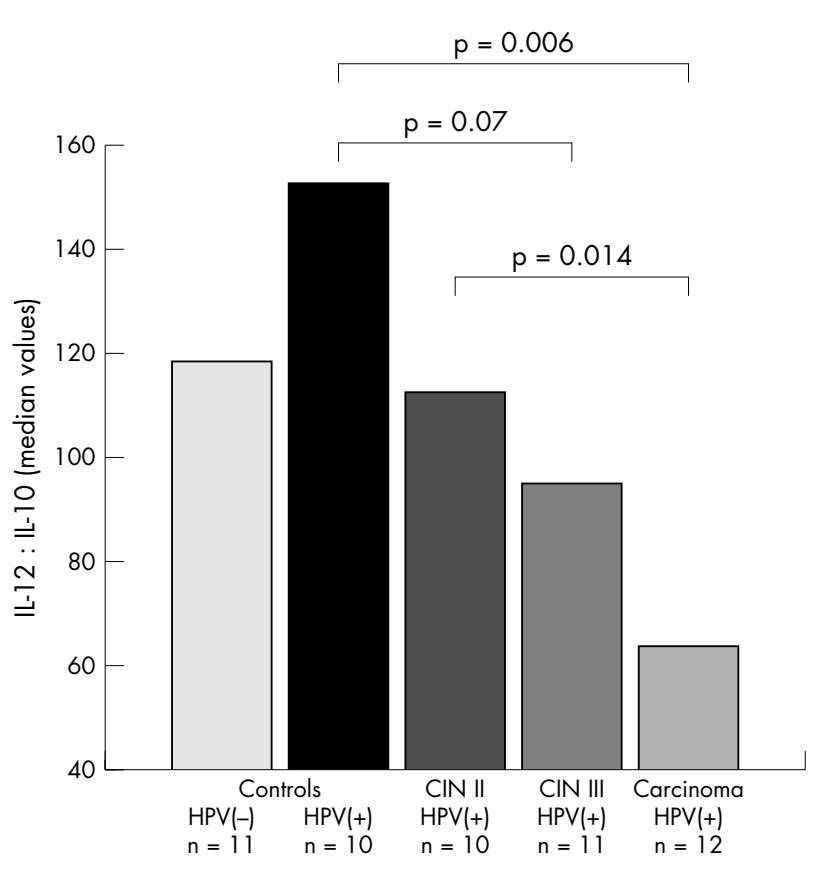

Figure 4 IL-12: IL-10 ratio in plasma of high risk HPV positive patients with cervical dysplasia and in high risk HPV negative and high risk HPV positive controls. CIN, cervical intraepithelial neoplasia; HPV, human papillomavirus; IL, interleukin.

groups. IL-4 concentrations were below the detection limit in most of the investigated plasma samples. IL-10 values were below the detection limit in most control women and women with moderate dysplasia, but were detectable in four of 11 patients with CIN III and seven of 12 invasive carcinomas. Table 2 shows the data for IL- 4 and IL- 10 .

A possible shift to increased expression of Th2-type cytokines in patients with carcinoma was evaluated by calculating the ratio of Thl-type cytokines IL-12, IFN $\gamma$, TNF $\alpha$, and IL-2 to the Th2-type cytokine IL-10 for all study groups. These ratios decreased significantly $(p<0.01$ for IFN $\gamma:$ IL-10, $p=0.02$ and $p<0.01$ for TNF $\alpha$ : IL-10, $p=0.03$ and $p=0.06$ for IL-2 : IL-10) when patients with carcinoma were compared with HR-HPV positive controls or patients with CIN II. Figure 4 shows as an example the ratio of IL-12 : IL-10.

\section{DISCUSSION}

Our data for the differential white blood cell counts in our patients are in agreement with the results of Onsrud et al, who reported increased leucocyte and neutrophil counts in cervical carcinoma, probably induced by inflammation and necrosis, and a decrease in absolute lymphocyte numbers in comparison with healthy controls. ${ }^{24}$ Studies by Gemignani et al and Balaram et al confirm these results. ${ }^{25}{ }^{26}$ Stratification of cytokine data for each $10^{6}$ leucocytes seems justified.

Our study showed a significant decrease in circulating IFN $\gamma$ concentrations in women with severe dysplasia and invasive carcinoma. IFN $\gamma$, secreted by Thl cells, cytotoxic T cells, and stimulated natural killer cells, is a major contributor to an effective Thl-type cellular immune response against HPV infections. ${ }^{7}$ Defective IFN $\gamma$ production may be associated with persistent HPV infection and the development of HPV related neoplasia. ${ }^{27}$ Our data for circulating IFN $\gamma$ agree with these reports.

TNF $\alpha$ has been shown to act synergistically with IFN $\gamma$ on cervical cancer cells by inducing apoptosis or necrosis. ${ }^{28}$ The biological effects of TNF $\alpha$ are mediated by its two receptors
TNFRI and TNFRII. Both receptors are present on many cell types and tissues; TNFRI is strongly expressed on cells that are sensitive to the cytotoxic actions of TNF $\alpha$, whereas TNFRII is strongly expressed on stimulated $\mathrm{T}$ and B cells. ${ }^{29}$ Several agents including $\mathrm{TNF} \alpha$ itself downregulate cell surface expression of both receptors by proteolytic cleavage of the extracellular domains that are shed as soluble proteins-sTNFRI and sTNFRII-into the circulation. Measurement of both soluble receptors, especially of sTNFRII, is often used to assess Thl-type activity in cell mediated immune responses. ${ }^{29-31}$ Our study showed no significant differences in TNF $\alpha$ values between study groups. However, there was a decrease in both soluble receptor concentrations in the plasma of patients with carcinoma, which reached significance for sTNFRII. This result reflects a decrease in circulating $\mathrm{TNF} \alpha$ and is in accordance with a disturbed Thl-type immune response after progression to CIN III and invasive carcinoma.

There were no significant differences in peripheral concentrations of the Thl-type cytokine IL-2 between our study groups, except a slight increase $(p=0.037)$ between HRHPV negative and HR-HPV positive controls, which could indicate an inflammatory response to the viral infection.

\section{"Interleukin 10 concentrations increased significantly with the severity of dysplasia"}

A possible shift to Th2-type cytokines in the course of development of cervical neoplasia may be reflected by increased plasma concentrations of Th2-type cytokines IL-4 and IL-10. However, we found no significant differences in IL-4 concentrations between our study groups. IL-10 concentrations increased significantly with the severity of dysplasia. IL-10 is a potent immunosuppressive cytokine, which inhibits $\mathrm{T}$ cell activation and Thl cell differentiation. This shift to Th2-type activity was characterised by Jacobs et al as a significant decrease in the IL-12: IL-10 ratio in patients with high grade squamous intraepithelial lesions. ${ }^{20}$ IL-12, a key cytokine in the induction of cell mediated immunity, promotes $\mathrm{T}$ cell differentiation into IFN $\gamma$ producing Thl cells and induces IFN $\gamma$ production in NK cells. The significant decrease in IL-12, IFN $\gamma, \mathrm{TNF} \alpha$, and IL-2 relative to IL-10 in patients with CIN III and carcinoma, as seen in our study, supports and extends the finding of Jacobs et al of a disturbed cellular immune response in the development of severe cervical dysplasia and cervical carcinoma. The presence of diseases that could influence circulating cytokine concentrations was excluded in all participants, so that presumably these results are the consequence of HPV infection.

The changes that we found within the peripheral cytokine network in cervical dysplasia and invasive cancer were not

Take home messages

- We found significant decreases in circulating interferon $\gamma$ and tumour necrosis factor $\alpha$ receptor $\|$ and an increase in interleukin 10 concentrations through the different stages of dysplasia to invasive carcinoma

- These results indicate a shift towards a T helper 2-type (Th2) cytokine pattern during the carcinogenesis of cervical cancer in women with cervical intraepithelial neoplasia (CIN) III lesions

- Vaccinations aimed at converting the Th2 response into a Th1 response might be useful in the treatment of patients with CIN II and II 
always in agreement with published data for individual cytokines. ${ }^{12-20}$ In a study by Niwa et al, ${ }^{12}$ IFN $\gamma$ concentrations in plasma from patients with cervical cancer and controls were similar, whereas in a study by Lebrecht et al,,$^{13}$ IFN $\gamma$ concentrations were below the detection limit in all groups. Our results for plasma concentrations of TNFRI were in accordance with the results of Sheu et al, ${ }^{17}$ who found significantly lower serum concentrations of sTNFRI in patients with cervical cancer when compared with women with a benign disease, uterine myoma. However, they differ from the results of Malejczyk et al, ${ }^{18}$ in whose study patients with HPV-16 associated CIN I/II and CIN III lesions and with HPV-16/18 associated squamous cervical cancer were compared with healthy controls. There were no significant differences in receptor values between women with CIN lesions and controls, but both receptor concentrations increased significantly in patients with cervical cancer. Important factors that contribute to differences in results compared with earlier studies include selection criteria for controls and patients. In our study, we selected patients and controls according to their state of infection with HR-HPV. The single presence of low risk HPV type 70 in one woman with cervical carcinoma could be explained by HR-HPV DNA integrated via the $\mathrm{Ll}$ region in the cellular genome, which can be missed by the GP5+/6+ PCR. With the exception of the studies of Jacobs et al and Malejczyk et al, none of the above mentioned studies investigated the presence of HPV. ${ }^{18} 20$ Moreover, stratification for leucocyte numbers might also explain these discrepancies. Immunocompetent leucocytes are the main producers of circulating cytokines. In contrast to all other studies, we calculated our results according to the leucocyte count and not for each millilitre of plasma. Finally, analytical methods for the measurement of cytokines may differ. Some studies used plasma, others serum, a choice that influences the quantitative outcome (unpublished results, 2005). All studies used enzyme linked immunoassays, which were produced by different manufacturers. This implies the use of different antibody systems for cytokine binding and quantification with different sensitivities.

Our results indicate a change to a Th2 cytokine pattern already manifest in women with CIN III lesions. This provides the theoretical base of the clinical policy to treat all women with CIN III lesions. Furthermore, it might indicate that, in patients with cervical cancer, vaccinations should be directed towards conversion of the Th2 response into a Thl response, and that they may also be useful in women with CIN II lesions.

In conclusion, our study showed characteristic changes within the peripheral cytokine network during the course of HR-HPV infection through different stages of dysplasia to invasive carcinoma: a significant decrease in circulating IFN $\gamma$ and STNFRII and an increase in IL-10 concentrations. This indicates a shift towards a Th2-type immune response in the development of cervical cancer. The changes in cell mediated immunity become manifest in patients with CIN III, which is in line with the clinical observation that most CIN III lesions do not regress spontaneously, and may ultimately progress into invasive carcinoma if not properly treated. ${ }^{32}$

\section{Authors' affiliations}

A G Bais, I Beckmann, T J M Helmerhorst, Department of Obstetrics and Gynaecology, Erasmus University Medical Centre, PO Box 2040, 3000 CA Rotterdam, The Netherlands

J Lindemans, Department of Clinical Chemistry, Erasmus University Medical Centre

P C Ewing, Department of Pathology, Erasmus University Medical Centre C J L M Meijer, P J F Snijders, Department of Pathology, Vrije Universiteit Medical Centre, PO Box 7057, 1007 MB Amsterdam, The Netherlands

\section{REFERENCES}

1 Kiaer SK, Chakerian B, van der Brule AJC, et al. High-risk human papillomavirus is sexually transmitted: evidence from a follow-up study of virgins starting sexual activity. Cancer Epidemiol Biomarkers Prev 2001;10:101-6.

2 Walboomers JMM, Jacobs MV, Manos MM, et al. Human papillomavirus is a necessary cause of invasive cervical cancer worldwide. J Pathol 1999;189:12-19.

3 Helmerhorst TJM, Meijer CJLM. Cervical cancer should be considered as a rare complication of oncogenic HPV infection rather than a STD. Int J Gynecol Cancer 2002; 12:235-6.

4 Muñoz N, Bosch FX, de SanjoséS, et al for the International Agency for Research on Cancer Multicenter Cervical Cancer Study Group. Epidemiologic classification of human papillomavirus types associated with cervical cancer. N Engl J Med 2003;348:518-27.

5 Moscicki A-B, Ellenberg JH, Farhat S, et al. Persistence of human papillomavirus infection in HIV-infected and -uninfected adolescent girls: risk factors and differences by phylogenetic type. J Infect Dis 2004;190:37-45.

6 Nobbenhuis MAE, Walboomers JMM, Helmerhorst TJM, et al. Relation of human papillomavirus status to cervical lesions and consequences for cervicalcancer screening: a prospective study. Lancet 1999;354:20-5.

7 Wu TC, Kurman RJ. Analysis of cytokine profiles in patients with human papillomavirus-associated neoplasms. J Natl Cancer Inst 1997;89:185-6.

8 Spellberg B, Edwards JE. Typel/type 2 immunity in infectious diseases. Clin Infect Dis 2001;32:76-102.

9 Clerici M, Shearer GM. The Th1-Th2 hypothesis of HIV infection: new insights. Immunol Today 1994;15:575-81.

10 Clerici $M$, Merola $M$, Ferrario $E$, et al. Cytokine production patterns in cervical intraepithelial neoplasia: association with human papillomavirus infection. J Natl Cancer Inst 1997;89:245-50.

11 Clerici M, Shearer GM, Clerici E. Cytokine dysregulation in invasive cervical carcinoma and other human neoplasias: time to consider the TH1/TH2 paradigm. J Natl Cancer Inst 1998;90:261-3.

12 Niwa Y, Akamatsu H, Niwa H, et al. Correlation of tissue and plasma RANTES levels with disease course in patients with breast or cervical cancer. Clin Cancer Res 2001;7:285-9.

13 Lebrecht A, Hefler L, Tempfer C, et al. Serum cytokine concentrations in patients with cervical cancer: interleukin- 4 , interferon- $\gamma$, and monocyte chemoattractant protein-1. Gynecol Oncol 2001;83:170-1.

14 De Jaco P, Asselain B, Orlandi C, et al. Evaluation of circulating tumor necrosis factor- $\alpha$ in patients with gynecological malignancies. Int $J$ Cancer $1991 ; 48: 375-8$.

15 Sarandakou A, Phocas I, Sikiotis K, et al. Cytokines in gynecological cancer. Anticancer Res 1997; 17:3835-40.

16 Chopra V, Dinh TV, Hannigan EV. Circulating serum levels of cytokines and angiogenic factors in patients with cervical cancer. Cancer Invest 1998; 16:152-9.

17 Sheu BC, Lin HH, Chang DY, et al. The potential of serum levels of soluble tumor necrosis factor receptor I as a biochemical marker in cervical cancer. Br J Obstet Gynaecol 1997;104:1314-19.

18 Malejczyk M, Jóźwiak J, Osiecka A, et al. Serum levels of soluble tumor necrosis factor receptors in patients with benign and malignant HPVassociated anogenital lesions. Int J Cancer 1997;73:16-9.

19 Gupta MM, Jain R, Parashari A, et al. Circulating immune profile in patients with pre-cancer and cancer of the cervix: a cross sectional study among Indian women. Bull Cancer 1993;80:852-6.

20 Jacobs N, Giannini SL, Doyen J, et al. Inverse modulation of IL-10 and IL-12 in the blood of women with preneoplastic lesions of the uterine cervix. Clin Exp Immunol 1998;111:219-24.

21 van den Brule ACJ, Pol R, Fransen-Dahlmeijer N, et al. GP5+/6+ PCR followed by reverse line blot analysis enables rapid and high-throughput identification of human papilloma virus genotypes. J Clin Microbiol 2002;40:779-87.

22 Jacobs MV, Snijders PJF, van den Brule AJC, et al. A general primer GP5+/6+ mediated PCR-enzyme immunoassay method for rapid detection of 14 highrisk and 6 low-risk human papillomavirus genotypes in cervical scrapings. J Clin Microbiol 1997;35:791-5.

23 De Groote D, Zangerle PF, Gevaert Y, et al. Direct stimulation of cytokines (IL$1 \beta$, TNF- $\alpha$, IL-6, IL-2, IFN- $\gamma$, and GM-CSF) in whole blood. I. Comparison with isolated PBMC stimulation. Cytokine 1992;4:239-48.

24 Onsrud M, Grahm I, Gaudernack G, et al. Lymphoid cell distribution as prognostic factor in carcinoma of the uterine cervix. Acta Obstet Gynecol Scand 1992;71:135-9.

25 Gemignani M, Maiman M, Fruchter R, et al. CD4 lymphocytes in women with invasive and preinvasive cervical neoplasia. Gynecol Oncol 1995:59:364-9.

26 Balaram P, Radhakrishna Pillai M, Padmanabhan TK, et al. Immune function in malignant cervical neoplasia: a multiparameter analysis. Gynecol Oncol 1988;31:409-23

27 Scott $M$, Nakagawa M, Moscicki AB. Cell-mediated response to human papillomavirus infection. Clin Diagn Lab Immunol 2001;8:209-20.

28 Suk K, Chang I, Kim YH, et al. Interferon $\gamma($ IFN- $\gamma$ ) and tumor necrosis factor $\alpha$ synergism in ME-180 cervical cancer cell apoptosis and necrosis. J Biol Chem 2001;276:13153-9.

29 Diez-Ruiz A, Tilz GP, Zangerle R, et al. Soluble receptors for tumour necrosis factor in clinical laboratory diagnosis. Eur J Haematol 1995;54:1-8.

30 Porteu F, Hieblot $C$. Tumor necrosis factor induces a selective shedding of its p75 receptor from human neutrophils. J Biol Chem 1994;269:2834-40.

31 Bartholdy C, Nansen A, Maerker O, et al. Soluble tumor necrosis factor (TNF)-receptor levels in serum as markers of anti-viral host reactivity. Clin Exp Immunol 1999;116:299-306.

32 Östor AG. Natural history of cervical intraepithelial neoplasia: a critical review. Int J Gynecol Pathol 1993;12:186-92. 